\title{
L'UNION EUROPEENNE ET LA ZONE DU PACIFIQUE
}

\author{
Sylvie André *
}

L'Union européenne est présente, sous des formes diverses, dans la région Pacifique. Au-delà

du cadre de sa politique commerciale extérieure, elle a des accords d'association avec un certain nombre de territoires insulaires.

Ainsi, parmi les 20 pays et territoires d'outre-mer (PTOM) énumérés dans l'annexe I de la décision 91/482/CEE du Conseil du 25 juillet 1991, relative à l'association des pays et territoires d'outre-mer à la Communauté économique européenne ${ }^{1}$, cinq sont situés dans la zone Pacifique : quatre territoires français, Polynésie française, Nouvelle Calédonie et ses dépendances, îles Wallis et Futuna, terres australes et antarctiques françaises et une colonie britannique, Pitcairn.

Huit pays ACP ("Afrique, Caraỉbes, Pacifique ") appartiennent à la même zone géographique: Fidji, Kiribati, Papouasie Nouvelle Guinée, îles Salomon, Samoa occidentales, Tonga, Tuvalu, Vanuatu. Ces pays ont donc noué des relations d'association avec l'Union européenne dans le cadre des conventions de Lomé.

\section{LA POLITIQUE EXTÉRIEURE DE L'UNION EUROPÉENNE DANS LA RÉGION PACIFIQUE}

Cette politique est essentiellement une politique commerciale du ressort de la Communauté européenne qui s'est développée peu à peu depuis le Traité de Rome sur la base de l'article 113 permettant la conclusion "d'accords tarifaires et commerciaux" ainsi qu' "avec un ou plusieurs états ou organisations internationales". Dans ce cadre, qui est celui de la politique commerciale commune étendu aux autres politiques communes, le Conseil statue à la majorité qualifiée, et l'acte s'impose à la Communauté ainsi qu'aux Etats membres.

* Ancien élève de l'ENA, Professeur des Universités, Université Française du Pacifique.

1 JORF, L 263 du 19 septembre 1991. 
La mise en place progressive d'une politique extérieure et de sécurité commune (PESC) depuis 1987, qui n'est pas une politique intégrée et nécessite l'unanimité pour la prise de décision, a créé quelques difficultés. La CE s'appuie notamment sur l'article 235 du traité prévoyant la possibilité "d'actions nécessaires" pour "réaliser l'un des objets de la Communauté", afin de donner une interprétation extensive de ses compétences. En pratique ceci se traduit désormais par la signature d'accords mixtes (politique commerciale et PESC). Les accords signés dans le cadre de la PESC doivent être ratifiés par chacun des états membres avant d'être mis en œuvre, ce qui en retarde l'application.

En terme de volume d'échanges de biens, de services et d'investissements, l'Union européenne est le premier partenaire économique de l'Australie. Des accords de coopération ont été conclus dans les domaines de la science et de la technologie, de la coopération industrielle, de la coordination de l'aide au développement dans la région du Pacifique ainsi qu'en matière d'énergie et d'environnement.

Par ailleurs le dialogue politique s'est renforcé avec la programmation de deux réunions au niveau ministériel par an entre les deux parties ainsi que de rencontres régulières entre parlementaires européens et australiens.

Avec la Nouvelle-Zélande, la base de la coopération est constituée par des accords préférentiels axés sur les produits agricoles. Ainsi, pendant de nombreuses années, les importations de beurre et d'agneau de Nouvelle-Zélande ont-elles bénéficié d'un accès préférentiel au marché commun. En 1991, a été signé un accord de coopération scientifique et technique dans les domaines de l'agriculture, de la biomasse, de la biotechnologie, de l'environnement, de la sylviculture, des énergies renouvelables ainsi que des technologies de l'information. ${ }^{2}$

\section{L'AIDE AU DÉVELOPPEMENT DES ETATS ACP DANS LA ZONE DU PACIFIQUE}

\section{A Les bases juridiques}

Reposant à l'origine sur une association octroyée sur la base de la quatrième partie du traité de Rome, les accords d'association se sont ensuite développés sur la base de l'article 238 du traité modifié par le traité de Maastricht afin de tenir compte de l'accession progressive à l'indépendance des pays africains :

La Communauté peut conclure avec un ou plusieurs Etats ou organisations internationales des accords créant une association caractérisée par des droits et obligations réciproques, des actions en commun et des procédures particulières .

2 Politiques de l'Union européenne-Relations extérieures, http : / / europa.eu.int, 3/03/1998. 
Cette association négociée et contractuelle s'est traduite par les deux conventions de Yaoundé entre la CEE et les Etats africains et malgache. ${ }^{3}$ La convention de Yaoundé prévoyait la possibilité pour les "Etats dont la structure économique et la production sont comparables à celles des Etats associés" 4 d'adhérer à la convention.

Sur cette double base, les conventions d'association ont été ouvertes aux états africains anglophones puis aux états des Caraïbes et du Pacifique dont la liste a été dressée par le protocole $n^{\circ} 22$ annexé à l'Acte d'adhésion. Les 70 pays ACP ayant adhéré à la convention de Lomé IV ont tu leurs différences et leurs oppositions pour signer une convention multilatérale unique de coopération globale. Cette convention expire le 29 février 2000 et les négociations sur la future convention démarreront en septembre $1998 .^{5}$ Le Commissaire européen en charge de l'aide au développement a reçu mandat pour une adaptation approfondie des accords aux évolutions politiques, économiques et sociales des pays ACP.

\section{B Les conventions de Lomé}

Le principe de partenariat est au centre des conventions qui se sont succédées. La négociation globale, le dialogue avec chaque Etat $\mathrm{ACP}$, la contractualisation et la prévisibilité des ressources d'aide disponible sont à la base de toutes les conventions depuis 1975. Le partenariat s'est peu à peu institutionnalisé avec la mise en place d'un conseil des ministres constitué du Conseil de l'Union européenne, de la Commission et de membres des gouvernements des Etats ACP, d'un comité des ambassadeurs et d'une assemblée consultative.

Toutefois, des adaptations successives ont eu lieu en fonction de l'évolution des besoins ainsi que de la philosophie du développement, donnant à la convention de Lomé un caractère pionnier. En effet, elle a forgé des instruments permettant d'éviter les chocs économiques dus aux fluctuations brutales des marchés, de favoriser les exportations de ces pays, ou encore de subordonner l'aide à la démocratisation des systèmes politiques.

Ainsi, dans les années 1970 ont été mis en place des mécanismes de compensation des pertes de recettes d'exportation pour les secteurs d'activités dont les économies des Etats ACP sont fortement dépendantes. Ces mécanismes, STABEX et SYSMIN, ont été étendus par la suite aux PTOM. Le STABEX (fonds de stabilisation des recettes d'exportation) concerne une cinquantaine de produits de base. Ses modalités d'utilisation ont été profondément transformées à l'occasion de la convention de Lomé IV, signée en

31963 et 1969.

$4 \quad$ Article 58 § 2 et 3 .

$5 \quad$ "Sept jours Europe ", n² 289, 10 novembre 1997. 
décembre 1989. Par ailleurs, aucun remboursement ne sera exigé. Les fonds doivent compenser des pertes de recettes à l'exportation pour les agents économiques touchés par cette baisse ou être utilisés à des fins de diversification.

Le SYSMIN (facilités de financement spéciales pour les produits miniers créées en 1976) s'applique notamment à la production de bauxite, cobalt, cuivre, étain, fer, manganèse, phosphate et uranium. Des subventions sont accordées si les recettes représentent $15 \%$ des exportations totales du pays (10\% pour les Pays moins avancés : PMA) et si la baisse de production atteint 10\%. L'instauration de COM (cadre d'obligations mutuelles) a représenté une amélioration sensible car elle oriente l'utilisation des fonds vers un processus de réforme macro-économique et sectorielle. La lenteur des déboursements est cependant un handicap important. ${ }^{6}$

Chaque FED (fonds européen de développement) est attaché à l'exécution de chacune des conventions dans le cadre d'une programmation quinquennale des besoins prioritaires. Le VIIIème FED a défini des axes principaux tels que la formation, l'autosuffisance alimentaire, les infrastructures ou encore les investissements industriels. En 1995, le montant des aides était de 1,5 milliard d'écus dont 145 millions d'aide à l'ajustement structurel et 828,5 millions d'aides programmables ${ }^{7}$ dont les aides à projets. Alors que l'aide à projets a un impact relativement limité surtout dans le secteur agricole, l'appui à l'ajustement structurel, exclusivement sous forme de dons, permet à la Communauté de fournir un soutien efficace aux programmes de stabilisation, soutien dont l'approche est désormais faite sur la base de négociations paritaires.

Les préférences commerciales accordées aux Etats ACP par l'Union européenne obéissent à trois principes qui font leur originalité :

- $\quad$ l'aide est stable et prévisible , 10 ans pour Lomé IV,

- $\quad$ ces préférences sont décidées d'un commun accord, ne peuvent être modifiées unilatéralement par l'Union européenne,

- les préférences commerciales sont par définition non réciproques. L'accès généralisé et en franchise de droits est garanti aux produits manufacturés et transformés, y compris les textiles et les vêtements. Pour les produits agricoles, l'accès est contingenté mais les prix d'achat sont élevés et les règles d'origine relativement libérales.

6 Commission européenne, Livre vert sur les relations entre l'Union européenne et les pays ACP à l'aube du 21ème siècle, 1997.

7 Idem. 
Toutefois l'intérêt de ce système préférentiel s'érode en même temps que baissent les droits de douanes conformément à l'objectif de libéralisation du commerce mondial poursuivi par le GATT. Le conseil du GATT a d'ailleurs estimé que la convention de Lomé contrevenait au principe du libre échange et qu'elle était discriminatoire envers les autres pays moins avancés (PMA). L'Union européenne a obtenu une dérogation dont le principe est reconsidéré chaque année. De plus l'OMC a condamné en septembre 1997 le régime commercial préférentiel accordé aux pays ACP producteurs de bananes.

En relation avec la fin du conflit Est/Ouest, la Convention de Lomé IV a été l'un des premiers accords de coopération comportant une dimension politique explicite avec l'introduction d'une clause relative au respect des droits de l'homme. Cette clause a été étendue par l'article 5 de Lomé IV révisé en 1995 au respect des principes démocratiques, de l'état de droit et de la bonne gestion des affaires publiques. Le principe d'une programmation de l'aide en deux tranches, qui en limite le caractère automatique, a par ailleurs été retenu.

Enfin la Convention de Lomé IV permet de mettre en évidence des changements de priorité de l'aide au développement: appui au développement social (aide budgétaire, sécurisation des dépenses sociales), actions en faveur du développement durable, de la préservation de l'environnement, aides aux réformes institutionnelles, au renforcement de la société civile, au développement des capacités administrative.

Toutefois, le Livre vert sur les relations entre l'Union européenne et les pays ACP à l'aube $d u 21^{e ̀ m e}$ siècle conclut à l'insuffisance des adaptations successives et à la nécessité de réformes profondes de l'aide au développement de l'Union européenne.

\section{La nécessaire évolution de l'aide au développement de l'Union européenne}

L'évaluation globale de l'aide au développement des pays ACP contraint à conclure à une efficacité limitée. Alors qu'il y a vingt ans ces pays réalisaient 3\% des échanges internationaux, ils ne représentent désormais que 1,5\% du commerce mondial. Les préférences commerciales ne leur ont pas permis de diversifier leurs exportations de manière significative, ni de gagner des parts de marché dans les Etats membres.

Ces résultats mitigés ont mis en évidence l'importance des facteurs institutionnels et macro-économiques dans le développement, ainsi l'instabilité politique, la mauvaise gestion des affaires publiques jouent-elles un rôle important dans la stagnation économique de certains pays ACP. Il en est de même de l'instabilité des taux de change ou encore de politiques d'affectation des ressources inefficaces.

Par ailleurs la disparition de la politique des blocs remet en cause les équilibres géostratégiques. Les équilibres économiques mondiaux ont été, eux aussi, profondément transformés par la libéralisation mondiale des échanges, par l'émergence de nouveaux 
pays industrialisés, ainsi que par l'apparition progressive de stratégies économiques et commerciales régionales.

Ces deux facteurs conjugués, efficacité limitée et changements des conditions mondiales, conduisent à repenser les priorités et les formes de l'aide au développement de l'Union européenne.

Le Livre vert sur les relations entre l'Union européenne et les pays ACP à l'aube du $21^{\text {ème }}$ siècle met l'accent sur la diversité des pays ACP, tant au point de vue géographique, qu'économique et politique. Ainsi, les huit états du Pacifique forment-ils un ensemble, même s'il est hétérogène. La Papouasie Nouvelle Guinée représente $70 \%$ de la population totale, alors que Tuvalu compte à peine 9000 habitants. Le revenu par tête est de 710 USD au Kiribati, alors qu'il est de 2130 USD à Fidji. Toutefois ces états ont un certain nombre de points communs : le caractère insulaire et la petite taille de ces économies les rendent très vulnérables aux facteurs externes. Ils dépendent des échanges commerciaux et sont exposés à des catastrophes naturelles telles que les cyclones. Ils ont donc des problèmes spécifiques de transport et de communication ainsi que de préservation des ressources naturelles. Ces caractéristiques amènent la Commission européenne à considérer que leur intégration dans le commerce mondial dépend de leur capacité à nouer des liens avec les économies à croissance rapide du "Pacific Rim " avec l'appui de l'Australie et de la Nouvelle-Zélande. L'aide européenne leur permettrait dans ce cas d'éviter une dépendance trop étroite à l'égard des " puissances " régionales.

D'ores et déjà, la Papouasie Nouvelle Guinée participe depuis 1989 au processus de libéralisation engagé dans le cadre de l'APEC (Asia Pacific Economic Cooperation). Deux organisations régionales déjà anciennes sont amenées à redéfinir leur rôle depuis la signature du traité de dénucléarisation du Pacifique de Rarotonga par la France le 25 mars 1995. La Commission du Pacifique Sud créée en février 1947 rassemble 27 états ou territoires de la région et joue un rôle important d'expertise technique en matière de développement rural, de pêche... Elle a adopté un nouveau nom lors de la tenue de sa $37^{\text {ème }}$ conférence : Commission des îles du Pacifique (COMPAC), traduisant sa volonté de se concentrer sur les questions spécifiques aux territoires insulaires océaniens. Pour sa part, le Forum du Pacifique Sud, assemblée des chefs de gouvernements de 16 états indépendants et de territoires librement associés, né en 1971, doit recentrer ses objectifs, ${ }^{8}$ mais les TOM français participent déjà à certains de ces organismes satellites tels que la SOPAC (South Pacific Applied Geoscience Commission) ou le Conseil du tourisme du

8 Philippe Auberger, Rapport fait au nom de la Commission des finances sur le projet de loi de finances pour 1998, annexe ${ }^{\circ} 37$. 
Pacifique Sud. ${ }^{9}$ A travers ces organisations régionales s'effectue un rapprochement des PTOM avec les pays ACP ainsi qu'avec les grands pays de la zone.

L'accent mis sur l'intérêt d'une telle coopération n'est pas une façon pour l'Union européenne d'envisager une diminution de son aide au développement mais plutôt de mieux la cibler et d'en améliorer l'efficacité.

Le livre vert développe une double réflexion : sur le contenu des accords futurs ainsi que sur leurs modalités.

Le dialogue politique doit être accru dans le sens du respect des droits de l'homme et en rapport avec la politique européenne de prévention des conflits. La sélectivité de l'aide doit être améliorée en fonction des besoins mais aussi des choix institutionnels.

La politique de coopération pourrait s'orienter vers le développement du secteur privé, l'appui aux politiques sociales afin d'intégrer les populations pauvres à la vie économique. L'un des axes majeurs de la réforme envisagée serait l'aide institutionnelle sous la forme de restructuration des services publics, d'aide à la gestion budgétaire, d'accroissement des capacités en droit des affaires, de protection du consommateur, de l'environnement. La nécessité de cette aide est apparue flagrante à la lumière de l'évaluation des politiques menées jusqu'ici. Cette coopération serait modulable en fonction des évolutions constatées.

La coopération financière et technique se ferait de moins en moins sous forme d'aides à projets et de plus en plus sous forme d'appui aux réformes macro-économiques et aux politiques sectorielles, ainsi que d'aides directes aux budgets.

De plus la forme même de la convention de Lomé est fortement remise en question pour une meilleure efficacité et une meilleure adaptation.

Faut-il maintenir un accord multilatéral ou privilégier des accords régionaux ? Peuton envisager des formes mixtes?

Doit-on envisager des accords commerciaux séparés et différents selon la structure économique des pays ACP et prévoir des accords spécifiques PMA, incluant les PMA$\mathrm{ACP}$ ?

Comment améliorer la coordination des aides avec celle des pays membres ou encore avec les organismes internationaux tels ceux de Bretton Woods, l'Europe étant le principal bailleur d'aide au développement?

9 Yves Pimont, Les Territoires d' Outre-mer, PUF, 1994. 
La gestion des fonds doit-elle continuer à être conjointe, ce qui est une source de retard et de complication? Peut-on envisager une modulation, visant à responsabiliser les pays $\mathrm{ACP}$, en fonction des capacités locales de gestion?

Les mois à venir devraient être décisifs pour la réorientation de la relation Union européenne/ pays $\mathrm{ACP}$ et par là même pour la relation Union européenne/ pays et territoires associés dans le Pacifique, étant donné les nombreux points communs entre ces deux régimes, les progrès vers l'autonomie de gestion ainsi que l'accroissement des compétences des TOM français. Il faut souligner cependant la différence très importante des niveaux de vie et de développement entre la Polynésie française, la Nouvelle Calédonie d'une part et les pays ACP du Pacifique d'autre part.

\section{LE RÉGIME D'ASSOCIATION DES PTOM}

Parties intégrantes des Etats dont ils relèvent, ce qui est le cas des territoires français, colonie ou pays dont "un Etat membre assure les relations extérieures ", les PTOM sont liés à l'Union européenne par des accords parmi les plus avantageux. En effet, le régime de l'association des PTOM est un régime intermédiaire entre l'intégration et le régime appliqué aux pays tiers, en particulier les pays dits " ACP " et il est la conséquence des "relations particulières " qu'entretiennent ces territoires non européens avec certains états membres.

Ce sont la quatrième partie du traité de Rome du 25 mars 1957 ainsi que celle du traité de Maastricht, sans modification, qui définissent le cadre de l'association des pays et territoires d'Outre-mer à l'Union européenne dans leurs articles 131 à 136 bis.

Le but essentiel de l'association est "la promotion du développement économique et social des pays et territoires". L'article 132 précise les objectifs en matière d'échanges commerciaux, d'investissement communautaire et de droit d'établissement des ressortissants et des sociétés. Les principes sont clairs. En matière économique, le régime applicable à l'Etat européen avec lequel le PTOM entretient des relations particulières est étendu aux autres états membres en vertu du principe de non-discrimination. Ainsi, en Polynésie française, les marchandises en provenance de la CEE acquittent-elles les mêmes droits d'entrée que les marchandises en provenance de la métropole, droits inférieurs à ceux acquittés pour les marchandises en provenance des pays tiers. Parallèlement, les importations en provenance des PTOM, excepté le rhum, sont dispensées de droits de douane dans tous les Etats membres.

En matière d'établissement le principe est celui de l'application du droit communautaire, c'est à dire celui de la libre circulation et de la liberté d'établissement. ${ }^{10}$

10 Cf art 8, art 52 et suivants du Traité sur l'Union européenne (Maastricht). 
Notons qu'en contrepartie, les habitants des TOM français sont citoyens de l'Union : "Est citoyen de l'Union toute personne ayant la nationalité d'un état membre". ${ }^{11}$

Toutefois des conventions ultérieures doivent régler la liberté de circulation des travailleurs des états membres ou des PTOM en fonction des dispositions qui régissent "la santé publique, la sécurité publique et l'ordre public. " (art 135). Une convention relative aux modalités et à la procédure de l'association (art 136) notamment en ce qui concerne le droit d'établissement a été annexée au traité de Rome, permettant des "dispositions particulières" aux PTOM. Ces conventions requièrent l'unanimité des Etats membres.

Depuis, des décisions du Conseil ${ }^{12}$ viennent préciser les termes de l'association des PTOM. La dernière date du 25 juillet 1991. Elle a été signée pour 10 ans avec une révision prévue à mi-parcours. Cette révision rencontre des difficultés car l'un des états membres, en l'occurrence les Pays-Bas, s'oppose aux modifications proposées par la Commission sur le régime commercial et la règle de l'unanimité a empêché un accord.

\section{LE CONTENU DES DÉCISIONS SUCCESSIVES D'ASSOCIATION}

Depuis la première décision du 25 février 1964, le contenu des décisions, en matière financière comme de libre circulation, tend à se rapprocher davantage du régime d'association avec les pays tiers, surtout les pays ACP, que d'une intégration plus grande à l'Union européenne, en particulier sous la pression de l'obtention par les TOM français d'une plus grande autonomie d'administration et de gestion. Ainsi, la dernière décision reprend-elle de nombreux points de la convention de Lomé IV. Toutefois, le régime d'association des PTOM demeure un régime octroyé et non résultant d'un accord comme dans le cas des pays ACP. ${ }^{13}$

\section{A Un volet financier}

Comme les pays ACP, les PTOM bénéficient des concours financiers du Fonds européen de développement (FED), et plus particulièrement ceux qui sont considérés comme les moins développés, ce qui est le cas de Wallis et Futuna pour la France, ainsi que de prêts sur ressources propres de la BEI.

Le FED comporte deux parties distinctes : des dotations programmables (programme indicatif + dotation pour projets régionaux) dont la France effectue la répartition de la

11 Art 8.1. du traité de Maastricht. En revanche, les ressortissants des PTOM britanniques ne bénéficiant pas de la pleine citoyenneté britannique ("full British citizenship ") ne sont pas citoyens de l'Union européenne.

12 Première décision 64/349/CEE, du 25 février 1964.

13 Cf. Daniel Dormoy, Association des pays et territoires d'Outre-mer à la Communauté européenne, fascicule 743 (ed du Juris-classeur). 
masse globale accordée au titre des PTOM, des ressources non programmables constituées de prêts spéciaux (capitaux à risques) du FED, des ressources du STABEX, du SYSMIN, de bonifications d'intérêt sur les prêts BEI, d'aides d'urgence et d'aides à l'accueil des réfugiés.

Dans le cadre du VIIème FED, les PTOM français ont bénéficié de 40,2 millions d'écus sur les 86 millions d'écus de financement de projets et programmes ainsi que d'une partie des 11,5 millions d'écus de dotation pour les projets régionaux des PTOM. Le STABEX bénéficie plus particulièrement aux producteurs de coprah de la Polynésie française, alors que les ressources du SYSMIN sont utilisées par la Nouvelle-Calédonie dans le cadre de la production de nickel.

Lors de la révision à mi-parcours de la décision d'association, entérinée lors du Conseil européen de Cannes (juin 1995), les montants dévolus aux PTOM ont été augmentés. Les concours financiers globaux au titre du VIIIème FED sont portés à 165 millions d'écus $(+17.9 \%)$ et à 35 millions d'écus pour la BEI $(+40 \%)$. Toutefois ces ressources nouvelles sont inutilisables du fait du blocage sur le volet commercial de la décision d'association dont nous avons déjà parlé. ${ }^{14}$

Par ailleurs, malgré la mise en place de structures ad hoc, telles qu'un "ordonnateur du PTOM ", d'un délégué de la Commission européenne pour le Pacifique, de "payeurs délégués " dans les PTOM et les pays membres, les modalités d'utilisation des ressources communautaires sont complexes et les procédures rigides, aussi les taux d'utilisation de ces ressources se situent-ils entre 50 et $65 \%$ de la masse globale suivant les territoires, ce qui ne manque pas d'être décevant quand on connaît leurs besoins.

Cependant, il est à noter que les ressources per capita affectés aux PTOM sont plus importantes que celles consacrées aux pays ACP alors que le volet financier est le même, et bien que la totalité soit infiniment supérieure. ${ }^{15}$

\section{B Un volet commercial instaure un système de préférence à l'importation dans la Communauté pour les produits originaires des PTOM}

La décision d'association de 1991 a mis en place un total libre accès des produits des PTOM au marché européen, ce qui n'est le cas que pour les produits industriels et certains produits agricoles des ACP. Aucune limitation quantitative n'était prévue

14 Notes et études documentaires 16 juin 1997, L'Avenir statutaire de la Nouvelle-Calédonie la documentation française, et Philippe Auberger, Rapport fait au nom de la Commission des finances sur le projet de loi de finances pour 1998, annexe $\mathrm{n}^{\circ} 37$.

15 Notes et études documentaires 16 juin 1997, L'Avenir statutaire de la Nouvelle-Calédonie, la documentation française. 
excepté pour le rhum. De telles facilités supposent que la CEE puisse contrôler l'origine des marchandises. La dernière décision d'association reprend à ce propos la définition $\mathrm{du}$ protocole $n^{\circ} 1$ annexé à la convention de Lomé IV : les produits doivent être entièrement obtenus ou suffisamment transformés dans les PTOM. Une telle disposition ne manque pas de susciter des problèmes en particulier, pour la zone Pacifique, en ce qui concerne les produits de la pêche, dont les prises des armements étrangers susceptibles d'être transformées sur place.

A l'inverse les droits d'entrée et les limitations quantitatives que peuvent instaurer les PTOM compte tenu des nécessités actuelles de leur développement ${ }^{16}$ ne doivent pas donner lieu à discrimination entre les différents états membres. Des mesures spécifiques visent à favoriser les échanges économiques entre PTOM ou avec les pays ACP.

Dans le cas de la Polynésie française, compte tenu de l'éloignement géographique, de la structure de l'économie dont les secteurs primaires et secondaires sont réduits, de la surévaluation du Franc Pacifique, ce volet commercial semble peu susceptible de développer des exportations vers les états membres et d'améliorer le taux de couverture.

\section{Un volet relatif aux règles d'établissement et de prestation de services des ressortissants, sociétés et entreprises des états membres dans les PTOM}

En la matière, les PTOM bénéficient d'un régime spécial en fonction de l'article 227, alinéa 3 du traité d'union européenne, défini aux articles 131-136.

Alors qu'en matière de libre circulation le droit communautaire est susceptible de s'appliquer, en fait, l'absence de convention prévue à l'article 135 du Traité de Maastricht ${ }^{17}$ en rend le principe inapplicable dans les PTOM. C'est en effet ce qu'ont conclu aussi bien le juge français que le juge communautaire. ${ }^{18}$

La décision d'association de 1964 a étendu la liberté d'établissement à la libre prestation de service. Il en résulte que sauf en cas de non-réciprocité, les ressortissants des états membres peuvent s'installer librement dans les PTOM. Ainsi la Cour de justice des communautés européennes a-t-elle condamné la France pour manquement parce que l'assemblée territoriale de Polynésie française de même que la Nouvelle Calédonie n'avaient pas pris les dispositions permettant l'application de ce principe. ${ }^{19}$ En 1995, la

16 Cf art 106 de la décision 91/482/CEE.

17 Cf ci-dessus.

18 CE, 12 mai 1989, Ministère de DOM-TOM c Piermont et arrêt de la cour de justice des communautés européennes du 12 décembre 1990, Kaefer et Procacci c Etat français.

19 Arrêt du 12 Décembre 1990, Commission c France. 
Commission a redemandé à la France de se mettre en conformité avec ce principe, faute de quoi elle saisirait à nouveau la Cour de justice. ${ }^{20}$

Pourtant, lors de la négociation de la dernière décision, la France avait obtenu que des dispositions spéciales soient prises conformément à l'article 136 du Traité. L'article 232 de la décision d'association du 25 juillet 1991 permet aux PTOM de demander à la Commission de Bruxelles d'établir des limitations au droit d'établissement des ressortissants des Etats membres, pour des secteurs déterminés et des périodes limitées dans le temps pour autant que de telles dispositions "soient limitées à de secteurs sensibles dans l'économie du PTOM concerné et s'inscrivent dans le but de promouvoir ou de soutenir l'emploi local".

Enfin, lors de la révision de la dernière décision d'association, la Commission européenne a proposé de rendre applicable aux ressortissants des PTOM ayant la citoyenneté européenne 22 programmes européens propres aux personnes tels Socrates, Leonardo, Tid, Media.

\section{Un volet institutionnel dénommé "le partenariat Commission/Etat membre/PTOM "}

Bien qu'à l'origine le régime d'association soit un régime octroyé, les PTOM français ont souvent participé à l'élaboration et à la mise en œuvre des décisions. L'évolution de leur statut a conduit à étoffer ce volet au cours des décisions successives. La décision d'association de 1991 a été élaborée à partir de réunions tripartites qui ont abouti à la proposition de la Commission au Conseil des ministres. Elle met en place un partenariat sur une base consultative, prévoyant la constitution de groupes de travail permanents ou spécifiques entre les trois partenaires. "Les avis des groupes de travail sont dûment pris en compte par la Commission" et "font en outre, le cas échéant, l'objet de propositions de la Commission...". ${ }^{21}$ La révision de la décision prévoit d'affermir ce partenariat.

Notons enfin que la Polynésie française dispose d'un représentant permanent auprès de la Communauté et que la France a institutionnalisé ce partenariat en soumettant les textes relatifs au FED à l'Assemblée territoriale de Polynésie française.

\section{L'AVENIR DU RÉGIME D'ASSOCIATION DES PTOM}

Le rapporteur spécial des crédits des TOM devant la commission des finances de l'assemblée nationale en 1996, M Gaston Flosse, par ailleurs président du gouvernement de la Polynésie française, avait exprimé ses craintes "que les apports attendus de

20 Réponse de J J Queyranne au Sénateur Daniel Millaud au Sénat, le 27 novembre 1997, cité in Tahiti Pacifique, janvier 1998.

21 Articles 234 à 236 de la décision du Conseil 91/482/CEE. 
l'association ne compensent pas les lourdes contraintes de l'intégration". 22 C'est bien là le problème central posé par le régime d'association des PTOM français auxquels s'applique une grande partie de la législation européenne.

Le gouvernement de la Polynésie française développe actuellement une réflexion tendant à mettre en évidence les contraintes des actuelles décisions d'association. D'une part la balance entre la perte financière induite par le régime préférentiel de droits d'entrée ${ }^{23}$ accordé aux états membres en fonction du principe de non-discrimination et les aides diverses octroyées par l'Union européenne est selon lui déficitaire. D'autre part, la réciprocité en matière de liberté d'établissement apparaît comme un frein à l'océanisation des cadres et comme un facteur de croissance du chômage des Polynésiens. Il est vrai que la crise économique qui sévit en Europe peut accélérer des mouvements migratoires qui s'étaient stabilisés. Un récent rapport du Conseil économique, social et culturel de la Polynésie française recommande de mettre en place des restrictions d'accès au marché du travail, qui, si elles sont théoriquement possibles dans le cadre de la dernière décision d'association, sont pratiquement difficiles à mettre en œuvre car elles sont contraires aux principes constitutionnels de l'égalité des citoyens et de la liberté d'aller et de venir sur le territoire de la République. ${ }^{24}$ En effet, le même régime devant être appliqué aux ressortissants métropolitains et aux ressortissants des états membres en vertu du principe européen de non-discrimination, l'établissement des français métropolitains serait lui aussi soumis à restriction. Il apparaît d'ailleurs que si pression il y a sur le marché du travail, elle est effectivement davantage du côté des français métropolitains que des Européens. ${ }^{25}$ Enfin, la Polynésie française a le sentiment que ses acquis statutaires en matière d'autonomie de gestion et de compétences sont menacés par la montée en puissance du droit communautaire dans l'ordre juridique national.

Lors de la conférence intergouvernementale de révision du traité de Maastricht convoquée en 1996, la France s'est fait le porte-parole des positions de ses territoires

22 Philippe Auberger, Rapport fait au nom de la Commission des finances sur le projet de loi de finances pour 1998, annexe $n^{\circ} 37$.

23 Les droits d'entrée constituent une des ressources principales du budget du Territoire de Polynésie française, qui, dans le cadre d'une intégration progressive à l'Union européenne devraient être peu à peu réduits. C'est dans cette perspective que l'on peut apprécier l'effort du gouvernement pour mettre en place une TVA (impôt à la consommation), susceptible de se substituer progressivement aux droits d'entrée.

24 Certains commentateurs font cependant état de précédents en matière de discrimination positive et envisagent même une réforme constitutionnelle permettant de restreindre la liberté d'établissement des français métropolitains et donc par voie de conséquence, des citoyens des états membres.

25 En 1996, 29 citoyens d'Etats membres travaillent en Polynésie contre environ 11600 métropolitains. 
d'Outre-mer. Le président de la République est intervenu sur le sujet lors de la conférence de Turin puis du Conseil européen de Dublin. La déclaration $n^{\circ} 36$ annexée au traité d'Amsterdam et adoptée le 17 juin 1997, prend en compte les remarques françaises et fixe un cadre satisfaisant pour un réexamen du régime d'association : 26

La conférence reconnaît que le régime spécial d'association des pays et territoires d'outre-mer (PTOM), résultant de la quatrième partie du traité instituant la Communauté européenne, a été conçu pour des pays et des territoires nombreux, de vaste superficie et à la population importante. Ce régime n'a que peu évolué depuis 1957.

La conférence observe qu'aujourd'hui, les PTOM subissent pour la plupart un retard structurel important, lié à des contraintes géographiques et économiques particulièrement handicapantes. Dans ces conditions, le régime spécial d'association tel qu'il a été conçu en 1957 ne peut plus répondre efficacement aux enjeux de développement des PTOM.

Après avoir énuméré quelques objectifs d'ordre général, le texte de la déclaration incite la Commission européenne à faire des propositions de réforme visant à une meilleure prise en compte de la diversité et de la spécificité de chaque PTOM, y compris en matière de liberté d'établissement ainsi qu'à une amélioration de l'efficacité de l'instrument financier aidant au développement économique et social des PTOM. Ces deux points vont dans le sens du mémorandum déposé par la France auprès de la Commission européenne au début de l'année 1997, proposant notamment la création d'un fonds particulier pour les PTOM, différent du FED, pour que des règles plus souples puissent être envisagées de même qu'une réévaluation éventuelle des crédits qui leur sont destinés.

Une réforme substantielle du régime d'association des PTOM devrait avoir lieu d'ici 1999.

La volonté de l'Union européenne de maintenir et éventuellement d'accroître son influence politique dans la région du Pacifique est indéniable. Cette influence passe par l'importance qu'elle accorde à l'aide au développement. Le nouveau millénaire, avec ses défis tels que la mondialisation et l'apparition de nouveaux équilibres économiques ou géostratégiques rend nécessaire une restructuration de cette aide.

Il est acquis qu'elle se fera grâce à une large concertation avec les partenaires traditionnels que sont les pays ACP du Pacifique, mais aussi avec les représentants des PTOM. Il semble que les décisions ou les accords d'association globaux, issus d'un processus historique de décolonisation, ne soient plus adaptés et que l'on s'achemine vers

26 Rodolphe Désiré, Avis présenté au nom de la commission des Affaires économiques et du Plan du Sénat sur le projet de loi de finances pour 1998, tome XXII. 
une plus grande différenciation des actes pour tenir compte de la diversité des situations des pays et territoires du Pacifique. Par ailleurs la prise en compte de la dimension régionale occupe une place importante dans la réflexion actuellement initiée par la Commission européenne.

\section{THE EUROPEAN UNION AND THE SOUTH PACIFIC}

In this paper Professor Andre reflects on the relationship between the European Union and the Associated States and Territories of the European Union which are situated in the Pacific: French Polynesia, New Caledonia, Wallis and Futuna, French Antarctic Territories, and Pitcairn. The other significant relationship of the European Union to the South Pacific area is through the Lome IV Convention. That treaty, signed in 1990 for ten years, has eight Pacific states as members: Fiji, Kiribati, Papua New Guinea, Solomon Islands, Samoa, Tonga, Tuvalu and Vanuatu.

The Lome Convention has been of significant financial and social benefit to the eight Pacific countries and, following the Green Paper on future relations between the European Union and the countries associated to it by the Lome treaty, preparations are now beginning for the re-negotiation of that relationship. The current treaty arrangement expires in February 2000. The Green Paper places emphasis on the differences between countries parties to the Lomé Treaty, and also on the importance of developing regional connections. This paper explores the bases of the Lomé arrangement, its special facilities for mining activities and sugar production, and the relationship of regional organisations to the aid from the European Union.

The associated territories of the European Union have a privileged status vis-à-vis the Union. That status is somewhere between that of full integration within a State member and the position of countries associated with the European Union through the Lomé Convention. The arrangements for these territories is to undergo substantial reform in 1999.

The conclusion of this paper is that the role of the European Union in the Pacific area will continue and increase. It is also suggested that new methods are now needed to deal with the relationship to the European Union, in order to reflect the diversity of the Pacific countries and territories and also to reflect, to a greater extent than at present, the regional dimension of this aspect of relations with the European Union. 
